\title{
Non-canonical propagation of high-order elliptic vortex beams in a uniaxially anisotropic medium
}

\author{
Fadeyeva T., Alexeyev C., Sokolenko B., Kudryavtseva M. and \\ Volyar A. \\ Physics Department, Taurida National V. I. Vernadsky University, \\ 4 Vernadsky Ave., 95007 Simferopol, Ukraine, e-mail: volyar@crimea.edu
}

Received: 27.01 .2011

\begin{abstract}
We have analysed propagation of nonparaxial and paraxial beams perpendicular to the optic axis in a uniaxially anisotropic medium (unbounded optically uniaxial crystal). We have presented the paraxial solutions in the form of generalised Hermite-Gaussian beams propagating perpendicular to the optic axis in a uniaxially anisotropic medium. We have also constructed the generalised Laguerre-Gaussian beams at the $z=0$ plane and analysed their evolution in a homogeneous isotropic medium. Comparing it with the evolution of standard Laguerre-Gaussian beams with $n=0$ and $m \neq 0$ in the crystal, we have revealed that the additional elliptic deformation of the extraordinary beam results in topological reactions that essentially distort the field structure for the account of different rotation rates of the vortex row originated from the centred degenerate optical vortex and the conoscopic pattern. We have predicted conversion of the vortex topological charge at the beam axis similar to that in astigmatic lenses and analysed the radical differences with this process. We have revealed synchronic oscillations of the spin angular momentum and the sign of the vortex topological charge at the beam axis.
\end{abstract}

Keywords: uniaxially anisotropic medium, optical vortex

PACS: 42.15.Dp, 42.25.Lc

UDC: 535.2

\section{Introduction}

As a rule, traditional description of a broad paraxial beam propagating orthogonally to the optic axis in crystals is restricted by the frameworks of plane-wave approach. Each plane wave is splintered into ordinary and extraordinary ones [1] at the crystal input. Since the phase velocities of these waves are different, the initial polarisation state of the wave is periodically transformed as the wave propagates along the crystal. More exact analysis has shown that the extraordinary Gaussian beam in crystals has an elliptic shape, which changes gradually when the beam is propagating, while the ordinary beam experiences only scale transformations [2-4].

A circularly polarised singular beam bearing the optical vortex [5] (or simply a vortex beam) brings its own correction into the propagation process. For example, the vortex beam whose axis is slightly tilted to the direction orthogonal to the optic axis of crystal 
rotates when the crystal rotates around the axis perpendicular to the optic axis. The optical vortex then rotates separately from the host beam, taking part in complex precessional and nutational motions. This unique property of the vortex allows one to create optical reducers for devices facilitating trapping, rotation and transportation of micro-particles [6]. Here the principal point is that the above processes do not break structural stability of the singular beam, i.e. the vortex composition of the beam is permanent when propagating. Such a fact has appeared strange from our point of view. Indeed, on one hand, different field distributions in the ordinary and extraordinary beams must inevitably result in spatial depolarisation of the beam; on the other hand, different scales along the $x$ - and $y$-axes must also yield distortion of the wavefronts of the partial beams, provoking phase perturbations in the vicinity of the vortex core in each circularly polarised component and inducing, ipso facto, transformations of the centred vortex composition.

Thus, the aim of this work is to analyse propagation of singular beams with elliptical cross sections bearing high-order optical vortices and to estimate structural transformations of the vortex core and the vortex beam as a whole.

\section{Basic equations and their solutions}

\subsection{Nonparaxial approach}

Let us consider the wave motion of a monochromatic, circularly polarised singular beam propagating perpendicular to the optic axis in an unbounded non-absorbing uniaxial crystal (i.e., uniaxially anisotropic medium - see Fig. 1) with the permittivity tensor

$$
\hat{\varepsilon}=\left(\begin{array}{ccc}
\varepsilon_{1} & 0 & 0 \\
0 & \varepsilon_{2} & 0 \\
0 & 0 & \varepsilon_{1}
\end{array}\right) .
$$

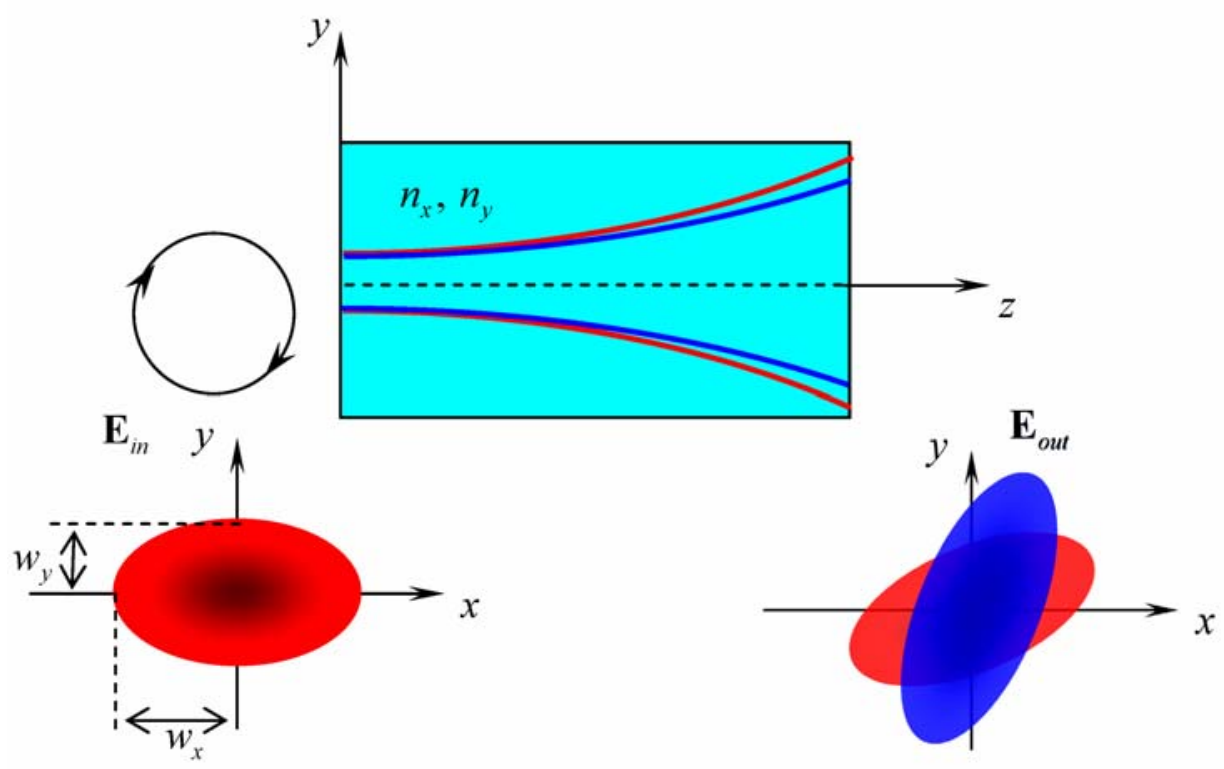

Fig. 1. Sketch of propagation of elliptic beam in optically uniaxial crystal. 
The Maxwell equations,

$$
\nabla \times \mathbf{E}=-i k \quad \mathbf{H}, \nabla \times \mathbf{H}=i k \quad \mathbf{D}, \mathbf{D}=\hat{\varepsilon} \mathbf{E},
$$

enable one to find solutions for the waves in the anisotropic medium with the material tensor given by Eq. (1) in the form of two beam types, $E$-beams and $H$-beams. Let us consider them separately.

\section{E-beams}

From Eqs. (2) one can write the wave equation for the electric field $\mathbf{E}$ in the form

$$
\nabla^{2} \mathbf{E}+k^{2} \hat{\varepsilon} \mathbf{E}=\nabla(\nabla \mathbf{E}),
$$

where $k$ stands for the wave number in the free space, as well as the equation

$$
\varepsilon_{1}\left(\partial_{x} E_{x}+\partial_{z} E_{z}\right)+\varepsilon_{2} \partial_{y} E_{y}=0 .
$$

The latter equation may be written as

$$
\nabla \mathbf{E}=-\frac{\varepsilon_{2}-\varepsilon_{1}}{\varepsilon_{1}} \partial_{y} E_{y} .
$$

Supposing the $E_{y}$ component to vanish ( $E_{y}=0$ ), we come to the equations

$$
\begin{aligned}
& \nabla^{2} E_{x}+k^{2} \varepsilon_{1} E_{x}=0, \nabla^{2} E_{z}+k^{2} \varepsilon_{1} E_{z}=0, \\
& \partial_{x} E_{x}=-\partial_{z} E_{z} .
\end{aligned}
$$

Eq. (7) reduces to the identity if

$$
E_{x}=\partial_{z} \Phi_{E}, E_{z}=-\partial_{x} \Phi_{E},
$$

while Eqs. (6) may be reduced to the single scalar Helmholtz equation for the function $\Phi_{E}$ :

$$
\nabla^{2} \Phi_{E}+k^{2} \varepsilon_{1} \Phi_{E}=0 .
$$

Finally, we can trace out the solutions of the Maxwell equations for the $E$-beams (or the ordinary beams) in the form

$$
\begin{aligned}
& E_{x}=\partial_{z} \Phi_{E}, \\
& E_{y}=0, \\
& E_{z}=-\partial_{x} \Phi_{E}, \\
& H_{x}=\frac{i}{k} \partial_{x y}^{2} \Phi_{E}, \\
& H_{y}=\frac{i}{k}\left(\partial_{z}^{2}+\partial_{x}^{2}\right) \Phi_{E}, \\
& H_{z}=-\frac{i}{k} \partial_{y z}^{2} \Phi_{E} .
\end{aligned}
$$

In fact, the solutions given by Eqs. (10)-(15) represent the vector field of nonparaxial beam propagating in a homogeneous isotropic medium with the refractive index $n_{x}=\sqrt{\varepsilon_{1}}$. 


\section{H-beams}

From Eqs. (2) we can also write out the wave equation for the magnetic field $\mathbf{H}$ in the form

$$
\begin{aligned}
& \nabla^{2} \mathbf{H}+k^{2} \varepsilon_{1} \mathbf{H}=-\left(\varepsilon_{1}-\varepsilon_{2}\right) \quad\left(\mathbf{e}_{\mathbf{x}} \partial_{z}-\mathbf{e}_{z} \partial_{x}\right) \quad\left(\partial_{z} H_{x}-\partial_{x} H_{z}\right), \\
& \partial_{x} H_{x}+\partial_{z} H_{z}+\partial_{y} H_{y}=0 \text {. }
\end{aligned}
$$

Assuming the $H_{y}$ component to vanish $\left(H_{y}=0\right)$, we obtain

$$
\begin{aligned}
& \nabla^{2} H_{x}+k^{2} \varepsilon_{1} H_{x}=-\frac{\left(\varepsilon_{1}-\varepsilon_{2}\right)}{\varepsilon_{2}} \quad \partial_{z}\left(\partial_{z} H_{x}-\partial_{x} H_{z}\right), \\
& \nabla^{2} H_{z}+k^{2} \varepsilon_{1} H_{z}=\frac{\left(\varepsilon_{1}-\varepsilon_{2}\right)}{\varepsilon_{2}} \quad \partial_{x}\left(\partial_{z} H_{x}-\partial_{x} H_{z}\right),
\end{aligned}
$$

together with

$$
\partial_{x} H_{x}=-\partial_{z} H_{z}
$$

Substituting Eq. (20) into Eqs. (18) and (19), we find that

$$
\begin{aligned}
& \partial_{x}^{2} H_{x}+\frac{\varepsilon_{2}}{\varepsilon_{1}} \partial_{y}^{2} H_{x}+\partial_{z}^{2} H_{x}+k^{2} \varepsilon_{2} H_{x}=0, \\
& \partial_{x}^{2} H_{z}+\frac{\varepsilon_{2}}{\varepsilon_{1}} \partial_{y}^{2} H_{z}+\partial_{z}^{2} H_{z}+k^{2} \varepsilon_{2} H_{z}=0 .
\end{aligned}
$$

These equations are consistent provided that

$$
H_{x}=\partial_{z} \Phi_{H}, H_{z}=-\partial_{x} \Phi_{H},
$$

while the scalar function $\Phi_{H}$ obeys the equation

$$
\partial_{x}^{2} \Phi_{H}+\frac{\varepsilon_{2}}{\varepsilon_{1}} \partial_{y}^{2} \Phi_{H_{x}}+\partial_{z}^{2} \Phi_{H}+k^{2} \varepsilon_{2} \Phi_{H}=0
$$

Thus, the electric and magnetic fields of the $H$-beams (or extraordinary beams) are

$$
\begin{aligned}
& E_{x}=-\frac{i}{k} \partial_{x y}^{2} \Phi_{H}, \\
& E_{y}=-\frac{i}{k}\left(\partial_{z}^{2}+\partial_{x}^{2}\right) \Phi_{H}, \\
& E_{z}=\frac{i}{k} \partial_{y z}^{2} \Phi_{H}, \\
& H_{x}=\partial_{z} \Phi_{H}, \\
& H_{y}=0, \\
& H_{z}=-\partial_{x} \Phi_{H} .
\end{aligned}
$$

In other words, the nonparaxial $H$-beam propagates through the uniaxial crystal as if through a homogeneous isotropic medium with the refractive index $n_{y}=\sqrt{\varepsilon_{2}}$. However, such a medium has a particular property: it reveals different scales along the $x$ - and $y$-axes 
(namely, $\Phi_{H}=\Phi_{H}(\bar{x}, \bar{y}, z)$, so that $\bar{x}=x$ and $\bar{y}=\frac{n_{x}}{n_{y}} y$ ). This circumstance enables us to use the results obtained in Ref. [7] for nonparaxial ordinary and extraordinary beams that propagate along homogeneous isotropic media, taking into account the refractive indices $n_{x}$ and $n_{y}$ and also different space scaling for the extraordinary beam. Nevertheless, we will focus our attention on the paraxial vector beams, laying aside the nonparaxial approach.

\subsection{Paraxial approximation for the $E$ - and $H$-beams}

Let the beam propagates along the $z$-direction in such a way that $\Phi_{E, H}=\Psi_{E, H}(x, y, z) \exp \left(i k n_{x, y} z\right)$ and the demand $\left|\partial_{z}^{2} \Psi\right|<<k\left|\partial_{z} \Psi\right|$ is fulfilled. Then Eqs. (9) and (24) for the $E$ - and $H$-beams are rewritten in the form

$$
\begin{aligned}
& \partial_{x}^{2} \Psi_{E}+\partial_{y}^{2} \Psi_{E}+i 2 k_{x} \partial_{z} \Psi_{E}=0, k_{x}=k n_{x}, \\
& \partial_{x}^{2} \Psi_{H}+\frac{n_{y}^{2}}{n_{x}^{2}} \partial_{y}^{2} \Psi_{H}+i 2 k_{y} \partial_{z} \Psi_{H}=0, k_{y}=k n_{y} .
\end{aligned}
$$

The electric and magnetic fields of the $E$ - and $H$-paraxial beams get the following forms.

E-beam:

$$
\begin{aligned}
& E_{x}=i k_{x} \Psi_{E} e^{i k_{x} z}, \\
& E_{y}=0, \\
& E_{z}=-\frac{k_{x}}{k} \partial_{x} \Psi_{E} e^{i k_{x} z}, \\
& H_{x} \approx 0, \\
& H_{y} \approx-i \frac{k_{x}^{2}}{k} \Psi_{E} e^{i k_{x} z}, \\
& H_{z} \approx \frac{k_{x}}{k} \partial_{y} \Psi_{E} e^{i k_{x} z} .
\end{aligned}
$$

H-beams:

$$
\begin{aligned}
& E_{x} \approx 0, \\
& E_{y} \approx-i \frac{k_{y}^{2}}{k} \Psi_{H} e^{i k_{y} z}, \\
& E_{z}=-\frac{k_{y}}{k} \partial_{y} \Psi_{H} e^{i k_{y} z}, \\
& H_{x}=i k_{y} \Psi_{H} e^{i k_{y} z}, \\
& H_{y}=0, \\
& H_{z}=-\partial_{x} \Psi_{H} e^{i k_{y} z} .
\end{aligned}
$$




\section{Elliptic beams}

\subsection{Basic relationships}

Since the initial circular cross section of the $H$-beam at the plane $z=0$ turns into the elliptical one when transmitting through the crystal $[3,4,6]$, we will find the solutions to the paraxial equations (31) and (32) in the form of elliptic beams. The theory of elliptic and astigmatic beams in free space has been developed and improved in a number of works (see, e.g., [8-14]). A general analysis of generalised Hermite-Gaussian and Hermite-Laguerre-Gaussian beams in the free space has been considered in the studies [1518]. Following Ref. 15, we will find a set of the generalised Hermite-Gaussian beams on the basis of generatrix functions as the particular solutions to Eqs. (31) and (32) in the form

$$
\begin{aligned}
& \Psi_{E}^{(0)}=\frac{w_{x x} w_{x y}}{\sqrt{\left(w_{x x}^{2}+i \zeta_{x}\right)\left(w_{x y}^{2}+i \zeta_{x}\right)}} \exp \left(-\frac{x^{2}}{w_{x x}^{2}+i \zeta_{x}}-\frac{y^{2}}{w_{x y}^{2}+i \zeta_{x}}\right), \\
& \Psi_{H}^{(0)}=\frac{w_{y x} w_{y y}}{\sqrt{\left(w_{y x}^{2}+i \zeta_{y}\right)\left(w_{y y}^{2}+i \zeta_{y}\right)}} \exp \left(-\frac{x^{2}}{w_{x y}^{2}+i \zeta_{y}}-\frac{\frac{n_{x}^{2}}{n_{y}^{2}} y^{2}}{w_{y y}^{2}+i \zeta_{y}}\right),
\end{aligned}
$$

where $w_{x x}, w_{x y}, w_{y x}, w_{y y}$ are waists of the $E$ - and $H$-beams at the $z=0$ plane (see Fig. 1 ) and $\zeta_{x, y}=\frac{2}{k_{x, y}} z$. Let us choose the differential operators in the form

$$
\begin{aligned}
& \hat{H}_{x, E}^{(m)}\left(\beta_{x, E}\right)=\left(\beta_{x, E}-i \zeta_{x}\right)^{m} \frac{\partial^{m}}{\partial x^{m}}, \hat{H}_{y, E}^{(m)}\left(\beta_{y, E}\right)=\left(\beta_{y, E}-i \zeta_{x}\right)^{m} \frac{\partial^{m}}{\partial y^{n}} \\
& \hat{H}_{y, H}^{(n)}\left(\beta_{y, H}\right)=\left(\beta_{y, H}-i \zeta_{y}\right)^{n} \frac{\partial^{n}}{\partial y^{n}}, \hat{H}_{y}^{(n)}\left(\beta_{y, H}\right)=\left(\frac{n_{y}}{n_{x}}\right)^{n}\left(\beta_{y, H}-i \zeta_{y}\right)^{n} \frac{\partial^{n}}{\partial \bar{y}^{n}}
\end{aligned}
$$

In a quite similar manner, we can construct the operators $\hat{H}_{y, E}^{(n)}\left(\beta_{y, E}\right)$ and $\hat{H}_{x, H}^{(m)}\left(\beta_{x, H}\right)$, where $\beta_{x, E, H}, \beta_{y, E, H}$ stand for the complex parameters and $\bar{y}=\frac{n_{x}}{n_{y}} y$. In particular, the operators have the important properties

$$
\lim _{\beta_{x} \rightarrow \infty}\left(\frac{1}{\beta_{x}} \hat{H}_{x}^{(m)}\right)=\frac{\partial^{m}}{\partial x^{m}}, \lim _{\beta_{y} \rightarrow \infty}\left(\frac{1}{\beta_{y}} \hat{H}_{y}^{(n)}\right)=\frac{\partial^{n}}{\partial y^{n}} .
$$

The operators given by Eqs. (47) and (48) commute with the operators $\hat{L}_{E}=\partial_{x}^{2}+\partial_{y}^{2}+i 2 k_{x} \partial_{z}$ and $\hat{L}_{H}=\partial_{x}^{2}+\partial_{\bar{y}}^{2}+i 2 k_{y} \partial_{z}$ of Eqs. (31) and (32), respectively (see [15]) and, consequently, with the functions $\Psi_{E}^{(m, n)}=\hat{H}_{E}^{(m, n)} \Psi_{E}^{(0)} \quad$ and 
$\Psi_{H}^{(m, n)}=\hat{H}_{H}^{(m, n)} \Psi_{H}^{(0)}$, where $\hat{H}_{E}^{(m, n)}=\hat{H}_{x, E}^{(m)} \hat{H}_{y, E}^{(n)}$ and $\hat{H}_{H}^{(m, n)}=\hat{H}_{x, H}^{(m)} \hat{H}_{y, H}^{(n)}$ are also the solutions to the paraxial equations (31) and (32). Thus, the solutions to these equations in the form of the generalised Hermite-Gaussian beams are as follows:

$$
\begin{gathered}
\Psi_{E}^{(m, n)}=i^{m+n}\left(\frac{\beta_{x, E}-i \zeta_{x}}{w_{x x}^{2}+i \zeta_{x}}\right)^{\frac{m}{2}}\left(\frac{\beta_{y, E}-i \zeta_{x}}{w_{x y}^{2}+i \zeta_{x}}\right)^{\frac{n}{2}} \times \\
\left.\left.H_{m}\left(x \sqrt{\frac{\beta_{x, E}+w_{x x}^{2}}{\left(\beta_{x, E}-i \zeta_{x}\right)\left(w_{x x}^{2}+i \zeta_{x}\right)}}\right) H_{n}\right) y \sqrt{\frac{\beta_{y, E}+w_{x y}^{2}}{\left(\beta_{y, E}-i \zeta_{x}\right)\left(w_{x y}^{2}+i \zeta_{x}\right)}}\right) \Psi_{E}^{(0)}, \\
\Psi_{H}^{(m, n)}=i^{m+n}\left(\frac{\beta_{x, H}-i \zeta_{y}}{w_{y x}^{2}-i \zeta_{y}}\right)^{\frac{m}{2}}\left(\frac{\beta_{y, H}-i \zeta_{y}}{w_{y y}^{2}-i \zeta_{y}}\right)^{\frac{n}{2}} \times \\
H_{m}\left(x \sqrt{\frac{\beta_{x, H}+w_{y x}^{2}}{\left(\beta_{x, H}-i \zeta_{y}\right)\left(w_{y x}^{2}+i \zeta_{y}\right)}}\right) H_{n}\left(\frac{n_{x}}{n_{y}} y \sqrt{\frac{\beta_{y, H}+w_{y y}^{2}}{\left(\beta_{y, H}-i \zeta_{y}\right)\left(w_{y y}^{2}+i \zeta_{y}\right)}}\right) \Psi_{H}^{(0)}
\end{gathered}
$$

where we have made use of the relationship

$$
\frac{\partial^{m}}{\partial x^{m}} e^{-x^{2}}=(-1)^{m} H_{m}(x) e^{-x^{2}} .
$$

In case if the parameters $\sqrt{\beta_{x, E}}=w_{x x}, \sqrt{\beta_{y E}}=w_{x y}, \sqrt{\beta_{x, H}}=w_{y x}, \sqrt{\beta_{y, H}}=w_{y y}$, the generatrix functions given by Eqs. (50) and (51) are transformed into standard HermiteGaussian beams:

$$
\begin{aligned}
& \Psi_{E}^{(m, n)}=i^{m+n}\left(\frac{w_{x x}^{2}-i \zeta_{x}}{w_{x x}^{2}+i \zeta_{x}}\right)^{\frac{m}{2}}\left(\frac{w_{x y}^{2}-i \zeta_{x}}{w_{x y}^{2}+i \zeta_{x}}\right)^{\frac{n}{2}} H_{m}\left(\sqrt{2} \frac{x}{\left|w_{x x}^{2}+i \zeta_{x}\right|}\right) H_{n}\left(\sqrt{2} \frac{y}{\left|w_{x y}^{2}+i \zeta_{x}\right|}\right) \Psi_{E}^{(0)} \\
& \Psi_{H}^{(m, n)}=i^{m+n}\left(\frac{w_{y x}^{2}-i \zeta_{y}}{w_{y x}^{2}+i \zeta_{y}}\right)^{\frac{m}{2}}\left(\frac{w_{y y}^{2}-i \zeta_{y}}{w_{y y}^{2}+i \zeta_{y}}\right)^{\frac{n}{2}} H_{m}\left(\sqrt{2} \frac{x}{\left|w_{y x}^{2}+i \zeta_{y}\right|}\right) H_{n}\left(\sqrt{2} \frac{\frac{n_{x}}{n_{y}} y}{\left|w_{y y}^{2}+i \zeta_{y}\right|}\right) \Psi_{H}^{(0)}
\end{aligned}
$$

If we have $\beta_{x, E} \rightarrow \infty, \beta_{y E} \rightarrow \infty, \beta_{x, H} \rightarrow \infty, \beta_{y, H} \rightarrow \infty$, Eqs. (49) transform the generatrix functions given by Eqs. (50) and (51) into elegant Hermite-Gaussian beams:

$$
\begin{aligned}
& \Psi_{E}^{(m, n)}=i^{m+n}\left(\frac{1}{\sqrt{w_{x x}^{2}+i \zeta_{x}}}\right)^{m}\left(\frac{1}{\sqrt{w_{x y}^{2}+i \zeta_{x}}}\right)^{n} H_{m}\left(\frac{x}{\sqrt{w_{x x}^{2}+i \zeta_{x}}}\right) H_{n}\left(\frac{y}{\sqrt{w_{x y}^{2}+i \zeta_{x}}}\right) \Psi_{E}^{(0)} \\
& \Psi_{H}^{(m, n)}=i^{m+n}\left(\frac{1}{\sqrt{w_{y x}^{2}+i \zeta_{y}}}\right)^{m}\left(\frac{1}{\sqrt{w_{y y}^{2}+i \zeta_{y}}}\right)^{n} H_{m}\left(\frac{x}{\sqrt{w_{y x}^{2}+i \zeta_{y}}}\right) H_{n}\left(\frac{\frac{n_{x}}{n_{y}} y}{\sqrt{w_{y y}^{2}+i \zeta_{y}}}\right) \Psi_{E}^{(0)}
\end{aligned}
$$




\subsection{Generalised elliptic beams in isotropic media}

Let us consider at first some important properties of the generalised Hermite-Gaussian beams in a homogeneous isotropic medium, which have not been focused at in Refs. 1518. Let us form the function

$$
Q=\left(\frac{w_{y}}{w_{x}}\right) \frac{1+\left(\frac{\beta_{x}}{w_{x}^{2}}\right)}{1+\left(\frac{\beta_{y}}{w_{y}^{2}}\right)},
$$

with $w_{x x}=w_{x}, w_{y y}=w_{y}, \beta_{x, y, E}=\beta_{x, y}, \zeta_{x, y}=\zeta, z=0$ in Eq. (50). It combines together all of the three basic parameters $\left(\frac{w_{y}}{w_{x}}\right),\left(\frac{\beta_{x}}{w_{x}^{2}}\right)$ and $\left(\frac{\beta_{y}}{w_{y}^{2}}\right)$ of the generalised HermiteGaussian beam. The evolution of the beam cross section is shown in Fig. 2. When $\left(\frac{\beta_{x}}{w_{x}^{2}}\right)=1$ and $\left(\frac{\beta_{y}}{w_{y}^{2}}\right)=1$, the beam turns into the standard elliptic Hermite-Gaussian beam. It is the case that corresponds to the Hermite-Gaussian beams whose field distribution preserves along the $z$-axis up to the scale factor. All the other cases are accompanied by vanishing intrinsic edge dislocations out of the plane $z=0$. When increasing the parameter $\left(\frac{\beta_{y}}{w_{y}^{2}}\right)$ under the condition $\left(\frac{\beta_{x}}{w_{x}^{2}}\right)=1$ (i.e., the beam perturbation is following the line $\left.\left(\frac{\beta_{x}}{w_{x}^{2}}\right)=1\right)$, the edge dislocations along the $y$-axis vanish while those along the $x$-axis remain unchanged for $z \neq 0$. Vice versa, when the parameter $\left(\frac{\beta_{x}}{w_{x}^{2}}\right)$ increases under the condition $\left(\frac{\beta_{y}}{w_{y}^{2}}\right)=1$, the edge dislocations along the $x$-axis vanish while the dislocation pattern does not change along the $y$-axis. When $\left(\frac{\beta_{x}}{w_{x}^{2}}\right) \rightarrow 0$, but $\left(\frac{\beta_{x}}{w_{x}^{2}}\right) \neq 0$ and $\left(\frac{\beta_{y}}{w_{y}^{2}}\right)=$ const, the amplitude of the intermediate intensity oscillations decreases along the $x$-axis at the $z=0$ plane. When we have $\left(\frac{\beta_{y}}{w_{y}^{2}}\right) \rightarrow 0,\left(\frac{\beta_{y}}{w_{y}^{2}}\right) \neq 0$ and $\left(\frac{\beta_{x}}{w_{x}^{2}}\right)=$ const , the oscillations decay along the $y$-axis. Finally, the generalised Hermite-Gaussian beam is 
transformed into the elegant Hermite-Gaussian beam, when $\left(\frac{\beta_{x}}{w_{x}^{2}}\right) \rightarrow \infty$ and $\left(\frac{\beta_{y}}{w_{y}^{2}}\right) \rightarrow \infty$.

The ellipticity of the spots in the intensity distribution of the beam is specified exclusively by the ratio $\left(\frac{w_{y}}{w_{x}}\right)$ at the $z=0$ plane.

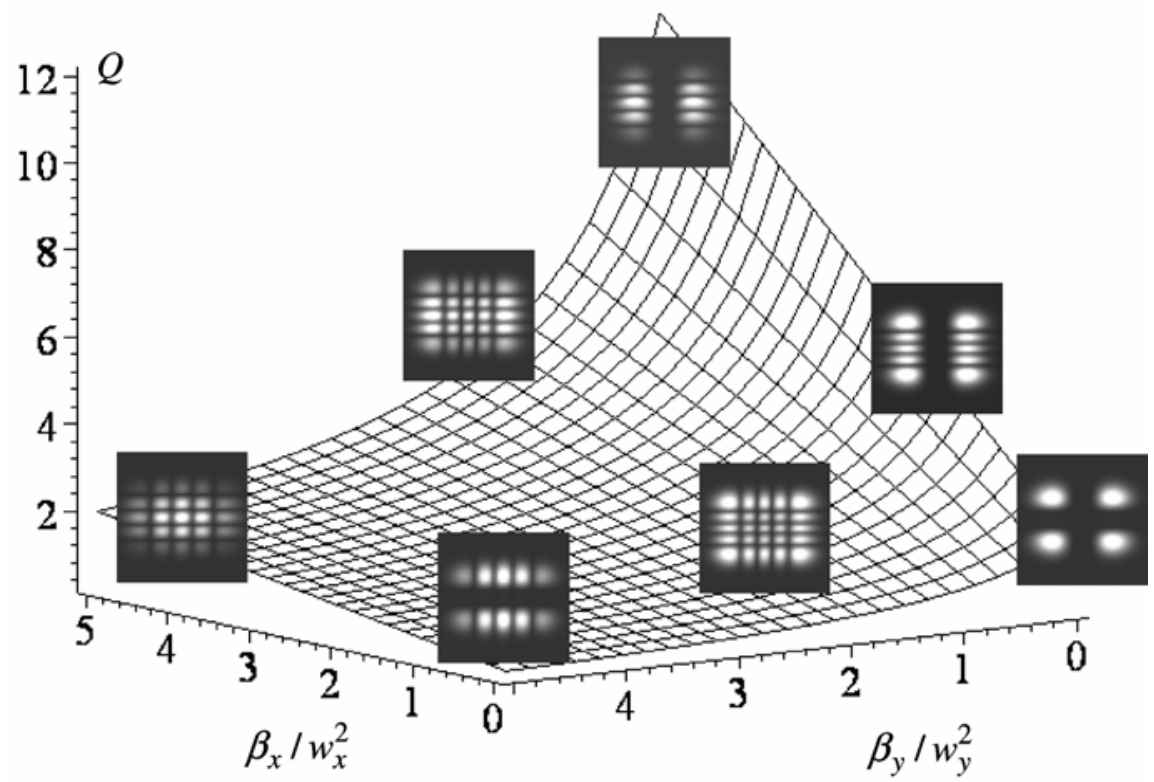

Fig. 2. Transformations of generalised Hermite-Gaussian beam $w_{x} / w_{y}=0.5, z=0$

Now let us form the generalised Laguerre-Gaussian (LG) beam and analyse its properties. For this aim we make use of the relation from Ref. 19 for the $z=0$ plane:

$$
\begin{aligned}
\Psi^{(m, n)}(z=0) & =(X+i Y)^{m} L_{n}^{m}\left(X^{2}+Y^{2}\right)= \\
& \frac{(-1)^{n+m}}{n ! 2^{2 n+m}} \sum_{p=0}^{n} \sum_{q=0}^{m}(-i)^{m+q}\left(\begin{array}{l}
n \\
p
\end{array}\right)\left(\begin{array}{c}
m \\
q
\end{array}\right) H_{2 p+q}(X) H_{2 n+m-2 p-q}(Y)
\end{aligned}
$$

In order to find the field distribution at the arbitrary $z$-plane, it is necessary that the Fresnel integral operator $\hat{F}$ act upon Eq. (58) [18] such that

$$
\begin{aligned}
& \Psi^{(m, n)}(x, y, z)=\hat{F}\left[\Psi^{(m, n)}(\xi, \eta, z=0)\right]= \\
& \frac{k}{2 \pi i z} \iint \exp \left(i \frac{k}{2 z}|\mathbf{r}-\mathbf{v}|^{2}\right) \Psi^{(m, n)}(\xi, \eta) d \mathbf{v}
\end{aligned},
$$

where $\mathbf{v}=\mathbf{e}_{\mathbf{x}} \xi+\mathbf{e}_{\mathbf{y}} \eta$. Using Eq. (50) for an isotropic medium, we come to

$$
\Psi^{(m, n)}(x, y, z)=\sum_{p=0}^{n} \sum_{q=0}^{m}(-i)^{m+q}\left(\begin{array}{l}
n \\
p
\end{array}\right)\left(\begin{array}{l}
m \\
q
\end{array}\right) H_{2 p+q}(\mathrm{X}) H_{2 n+m-2 p-q}(\Upsilon) \Psi^{(0)}(x, y, z)
$$


where

$$
\begin{gathered}
H_{2 p+q}(\mathrm{X})=\left(\sqrt{\frac{\beta_{x}-i \zeta}{w_{x}^{2}+i \zeta}}\right)^{2 p+q} H_{2 p+q}\left(x \sqrt{\frac{\beta_{x}+w_{x}^{2}}{\left(\beta_{x}-i \zeta\right)\left(w_{x}^{2}+i \zeta\right)}}\right), \\
H_{2 n+m-2 p-q}(\Upsilon)=\left(\sqrt{\frac{\beta_{y}-i \zeta}{w_{y}^{2}-i \zeta}}\right)^{2 n+m-2 p-q} H_{2 n+m-2 p-q}\left(y \sqrt{\frac{\beta_{y}+w_{y}^{2}}{\left(\beta_{y}-i \zeta\right)\left(w_{y}^{2}+i \zeta\right)}}\right),
\end{gathered}
$$

and the normalised factor in Eq. (60) has been omitted.

For the high-order optical vortex embedded into the LG beam with $n=0$, Eq. (58) is reduced to the form

$$
(X+i Y)^{m}=\sum_{q=0}^{m}(-i)^{m+q}\left(\begin{array}{c}
m \\
q
\end{array}\right) H_{q}(X) H_{m-q}(Y),
$$

so that the wave function is now transformed into

$$
\Psi^{(m, 0)}(x, y, z)=\sum_{q=0}^{m}(-i)^{m+q}\left(\begin{array}{l}
m \\
q
\end{array}\right) H_{q}(\mathrm{X}) H_{m-q}(\Upsilon) \Psi^{(0)}(x, y, z) .
$$

We have analysed the transformations of the standard elliptic LG beams with $\beta_{x}=w_{x}^{2}, \beta_{y}=w_{y}^{2}$. Typical evolutions along the z-axis of the intensity and the phase dis-

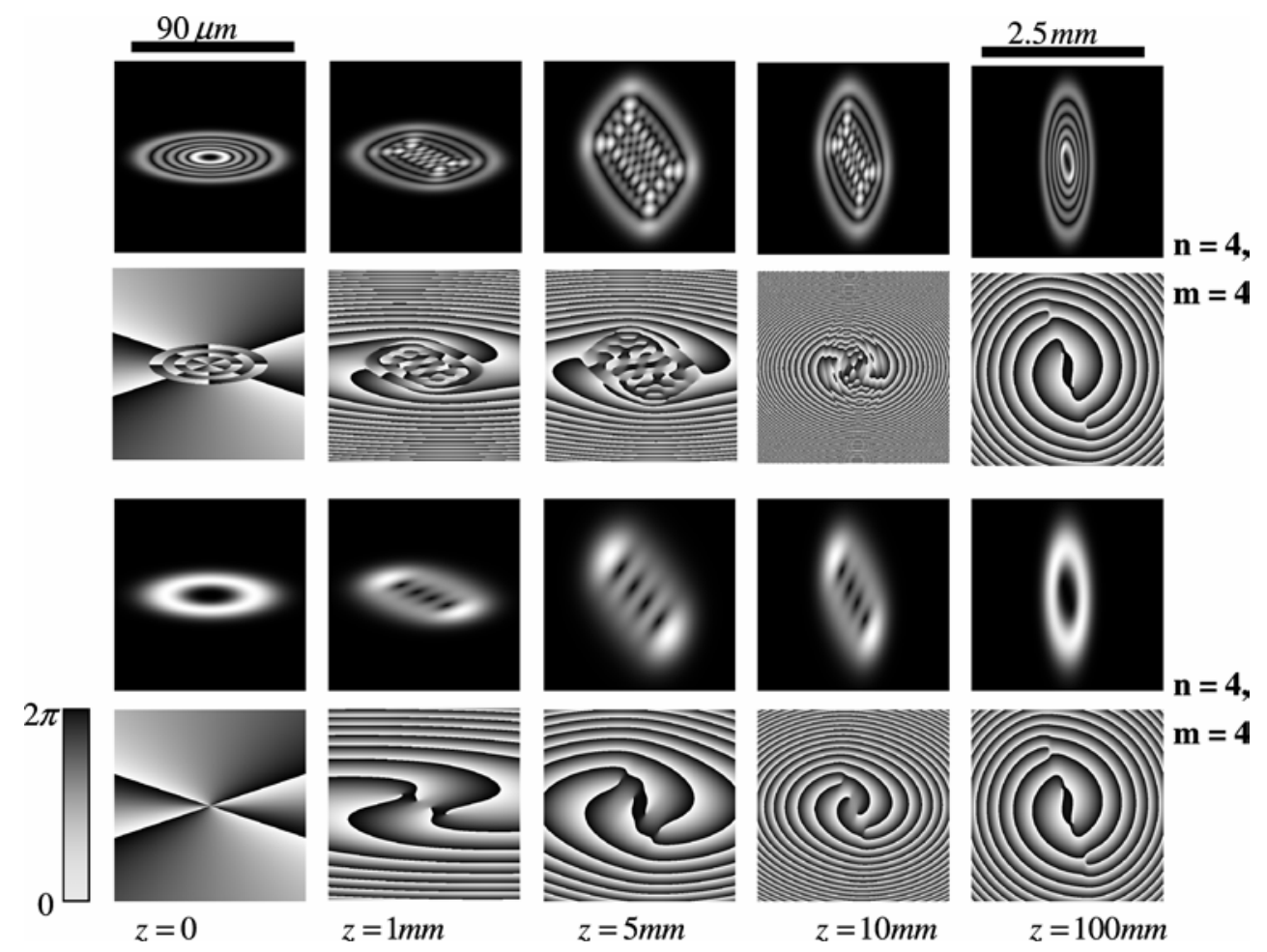

Fig. 3. Evolution along the $z$-axis of intensity and phase distributions in the elliptic beam with $w_{x}=30 \mu \mathrm{m}, w_{y}=10 \mu \mathrm{m}$ (the refractive index $n=2.3$ and the wavelength $\lambda=0.6328 \mu \mathrm{m})$. 
tributions in the elliptic LG beams with $n=4, m=4$ and $n=0, m=4$ are shown in Fig. 3 . Even a very small shift of the observation plane from the $z=0$ plane results in destroying the phase and intensity pattern as a whole in the both $\mathrm{LG}$ beams. However, the $\mathrm{LG}_{4,4}$ beam bearing both the four edge dislocations and the four-fold degenerate vortex forms a more complex pattern than that shaped by the $\mathrm{LG}_{0,4}$ beam with only the four-fold degenerate vortex. The elliptic perturbation out of the $z=0$ plane just draws up the high-order vortex into the row from four singly charged vortices, similar to that in the Ince-Gaussian beams $[11,21]$, the vortex row being synchronously rotated when the beam is propagating. A more complex picture of destroying phase singularities occurs in the elliptic $\mathrm{LG}_{44}$ beam. The destroying process affects not only the degenerate centred vortex but also the ring dislocations. As the beam propagates, the ring dislocations begin going to pieces, forming a complex cluster of optical vortices. At the far field, the singly charged vortices in the cluster gather together, recovering the ring dislocations. However, the degenerate vortex state at the axis does not restore, in contrast to those described in Refs. 18 and 21. Our computer simulations have shown that the distance between the vortices decreases relative to a whole scale of the beam cross section, though the vortices are not centred at the axis, at least at the distance up to $10 \mathrm{~m}$.

\subsection{Circularly polarised elliptic beam in the crystal}

The circularly polarised electric field of the elliptic beam is shaped as follows:

$$
E_{+}=E_{x}-i E_{y}, \quad E_{-}=E_{x}+i E_{y},
$$

where the polarisation components $E_{x}$ and $E_{y}$ are specified by Eqs. (33) and (40). In the normalised form they can be written as

$$
E_{x}=\Psi_{E} e^{i k_{x} z}, E_{y}=i \Psi_{H} e^{i k_{y} z},
$$

so that Eqs. (65) acquire the following form:

$$
E_{+}=\Psi_{E} e^{i k_{x} z}+\Psi_{H} e^{i k_{y} z}, \quad E_{-}=\Psi_{E} e^{i k_{x} z}-\Psi_{H} e^{i k_{y} z} .
$$

Here we will deal only with the case of the standard elliptic beams, with the complex amplitudes specified by Eqs. (53) and (54). In order that the elliptic beam have only one circularly polarised component at the $z=0$ plane (e.g., the $E_{+}$component: $\left.E_{-}(z=0)=0\right)$, it is necessary to match the initial beam parameters (namely, $\left.w_{x x}=w_{y x}=w_{x}, w_{x y}=w_{y}\right)$ so that $\Psi_{E}(x, y, z=0)=\Psi_{H}(x, y, z=0)$ :

$$
\begin{aligned}
& \Psi_{E}^{(m, n)}=\left(\frac{\sigma_{x x}^{*}}{\sigma_{x x}}\right)^{\frac{m}{2}}\left(\frac{\sigma_{x y}^{*}}{\sigma_{x y}}\right)^{\frac{n}{2}} H_{m}\left(\sqrt{2} \frac{x}{w_{x}\left|\sigma_{x x}\right|}\right) H_{n}\left(\sqrt{2} \frac{y}{w_{y}\left|\sigma_{x y}\right|}\right) \Psi_{E}^{(0)}, \\
& \Psi_{H}^{(m, n)}=\left(\frac{\sigma_{y x}^{*}}{\sigma_{y x}}\right)^{\frac{m}{2}}\left(\frac{\sigma_{y y}^{*}}{\sigma_{y y}}\right)^{\frac{n}{2}} H_{m}\left(\sqrt{2} \frac{x}{w_{x}\left|\sigma_{y x}\right|}\right) H_{n}\left(\sqrt{2} \frac{y}{w_{y}\left|\sigma_{y y}\right|}\right) \Psi_{H}^{(0)},
\end{aligned}
$$


where $\sigma_{x x}=1+i z / z_{x x}, \sigma_{x y}=1+i z / z_{x y}, \sigma_{y x}=1+i z / z_{y x}, \sigma_{y y}=1+i z / z_{y y}$,

$$
\begin{gathered}
z_{x x}=k_{x} w_{x}^{2} / 2, z_{y x}=k_{x} w_{y}^{2} / 2, z_{y x}=k_{y} w_{x}^{2} / 2, z_{y y}=\left(k_{x}^{2} / k_{y}\right) w_{y}^{2} / 2, \text { and } \\
\Psi_{E}^{(0)}=\frac{1}{\sqrt{\sigma_{x x} \sigma_{x y}}} \exp \left(-\frac{x^{2}}{w_{x} \sigma_{x x}}-\frac{y^{2}}{w_{y} \sigma_{x y}}\right), \\
\Psi_{E}^{(0)}=\frac{1}{\sqrt{\sigma_{y x} \sigma_{y y}}} \exp \left(-\frac{x^{2}}{w_{x} \sigma_{y x}}-\frac{y^{2}}{w_{y} \sigma_{y y}}\right) .
\end{gathered}
$$

Moreover, we will restrict ourselves only to consideration of the high-order vortex beams with $n=0$, whose transverse field components go over the form

$$
\begin{gathered}
E_{x}^{(m, 0)}=\Psi_{E}^{\{0\}} e^{i k_{x} z} \sum_{q=0}^{m}(-i)^{m+q}\left(\begin{array}{c}
m \\
q
\end{array}\right)\left(\frac{\sigma_{x x}^{*}}{\sigma_{x x}}\right)^{\frac{q}{2}}\left(\frac{\sigma_{x y}^{*}}{\sigma_{x y}}\right)^{\frac{m-q}{2}} H_{q}\left(\frac{\sqrt{2} x}{w_{x}\left|\sigma_{x x}\right|}\right) H_{m-q}\left(\frac{\sqrt{2} y}{w_{y}\left|\sigma_{x y}\right|}\right) \\
E_{y}^{(m, 0)}=i \Psi_{H}^{\{0\}} e^{i k_{y} z} \sum_{q=0}^{m}(-i)^{m+q}\left(\begin{array}{c}
m \\
q
\end{array}\right)\left(\frac{\sigma_{y x}^{*}}{\sigma_{y x}}\right)^{\frac{q}{2}}\left(\frac{\sigma_{y y}^{*}}{\sigma_{y y}}\right)^{\frac{m-q}{2}} H_{q}\left(\frac{\sqrt{2} x}{w_{x}\left|\sigma_{y x}\right|}\right) H_{m-q}\left(\frac{\sqrt{2} y}{w_{y}\left|\sigma_{y y}\right|}\right) .
\end{gathered}
$$

Transformations of the intensity distribution along the crystal length $z$ in such a vortex beam are illustrated by Fig. 4 . The difference of the wave numbers and the field distributions in the $E_{x}$ and $E_{y}$ components entails destructive interference that forms vortex dipoles breaking a straight row of the vortices originated from the centred degenerate singularity (see Fig. 3). At the same time, the details of the transformation process depend on difference of the ordinary $\left(n_{x}\right)$ and extraordinary $\left(n_{y}\right)$ refractive indices of the crystal.

A large difference $\Delta n=n_{x}-n_{y}$ results in different rotation angles of the vortex row in each of the linearly polarised components. Besides, any additional perturbation causes different deformations of the beam components along the $x$ - and $y$-axes. The contributions

a
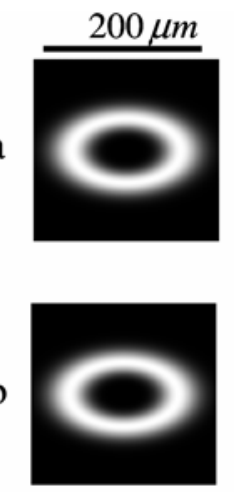

$z=0$


$z=5 \mathrm{~mm}$

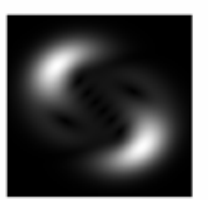

$z=15 \mathrm{~mm}$

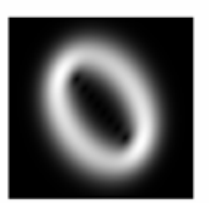

$z=25 \mathrm{~mm}$


Fig. 4. Transformation of intensity distributions in the $E_{+}$component of the vortex beam with $n=0$ and $m=8$ in the crystals with (a) $n_{x}=3, n_{y}=2$ and (b) $w_{x}=50 \mu \mathrm{m}, w_{y}=30 \mu \mathrm{m}$. 
of these processes to the far field manifest themselves in the form of a partial separation of the beam ellipses shown in Fig. $4 \mathrm{a}$ at the distance $z=1 \mathrm{~m}$. The beam overlapping is negligibly small for small values $\Delta n$ shown in Fig. $4 \mathrm{~b}$. In the far field, the basic vortices gather together near the beam axis as in Fig. 3 for the ordinary beam, without forming the centred degenerate vortex, while the additional vortices originated from the vortex dipoles annihilate with each other. Eventually, the elliptically deformed conoscopic pattern consisting of bright and dark hyperbolic lines is shaped both in the $E_{+}$and $E_{-}$ components.

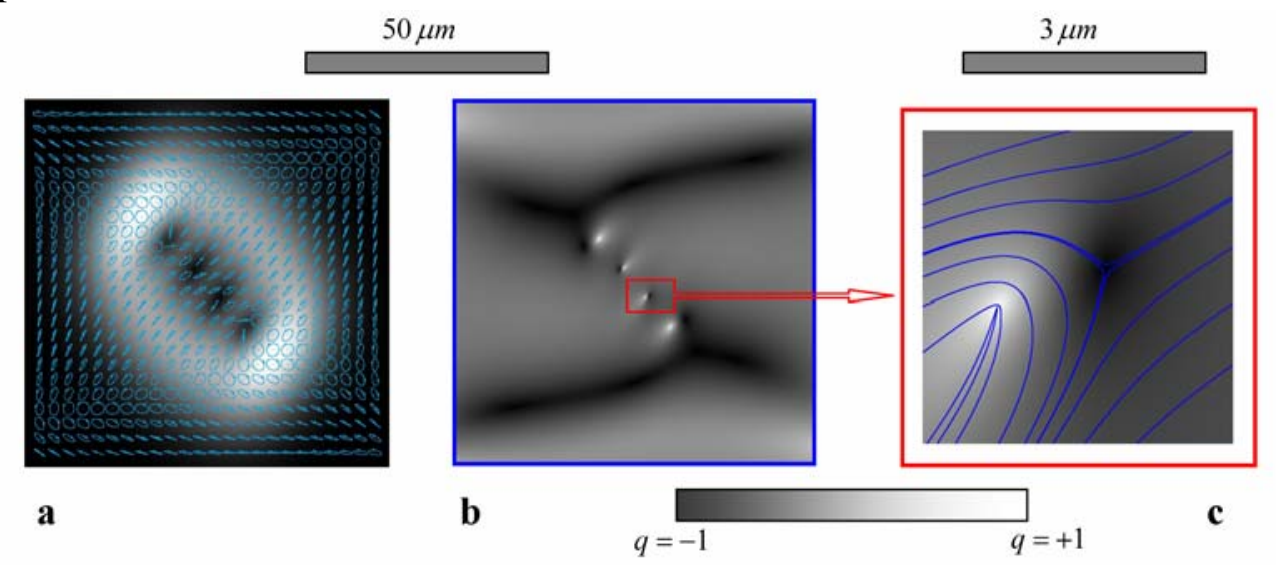

Fig. 5. Polarisation distributions in the vortex beam: (a) distribution of polarisation states on the background of intensity distribution of the vortex beam with $w_{x}=10 \mu \mathrm{m}, w_{y}=5 \mu \mathrm{m}, \quad n=0, m=4, \quad n_{x}=2.5, n_{y}=2$ and $z=1 \mathrm{~mm}$, (b) distribution of polarisation ellipticity $q$, and (c) stream lines of polarisation states on the background of ellipticity distribution.

The partial beam overlapping is tightly coupled with homogeneity of the polarisation states at the beam cross section. The polarisation inhomogeneity is associated with the vortex topological charge in the beam. The higher the vortex charge the stronger the polarisation inhomogeneity manifests itself. The patterns shown in Fig. 5 confirm this supposition. Indeed, the centres of the splintered singly charged vortices in the linearly polarised $E_{x}$ and $E_{y}$ components do not coincide with each other, forming a perturbed phase gradient in the vicinity of the vortex cores in the circularly polarised components. Fig. 5b illustrates distribution of the polarisation ellipticity $q= \pm b / a$ (with $b$ and $a$ being semi-axes of the polarisation ellipse). Interchange of the bright and dark spots on the map of ellipticity in Fig. 5b points out the sharp polarisation transformations in these beam areas. The most visual characteristics of the polarisation state distribution is the stream lines (the lines tangential to the major ellipse axis at each field point) [22]. The patterns of these lines shown in Fig. 5c represent a vector dipole formed by the coupled pair of the star and lemon [22]. When the elliptic beam propagates, the vector dipoles rotate around their mutual centres and roam about the beam cross section, changing radically the polarisation state of the beam as a whole. At the far field, the vector dipoles are 
drawn up together near the beam axis at the scale of the whole beam. In fact, the scale of the beam increases in leaps and bounds, when compared with the distance between the partial vortices. However, they cannot draw together at such a distance to annihilate, preserving some polarisation disorder at any crystal length.

\section{Spin angular momentum and conversion of the topological charge}

Let us consider in brief aftermath of inhomogeneity of the polarisation state over the beam cross section and a beam perturbation along the y-axis on the example of the loworder vortex beam. Our question is whether the centred vortex changes the sign of its topological charge when transmitting? To describe this process we rewrite the electric fields of the $E_{+}$and $E_{-}$components in Eqs. (71) and (72) for $n=0, m=1$ near the beam axis:

$$
E_{+}^{(1,0)} \approx A_{+} \frac{x}{w_{x}}+i a B_{+} \frac{y}{w_{y}}, E_{-}^{(1,0)} \approx A_{-} \frac{x}{w_{x}}+i a B_{-} \frac{y}{w_{y}}
$$

where

$$
\begin{aligned}
& A_{ \pm}=\frac{e^{i \beta_{x} z}}{\sigma_{x x} \sqrt{\sigma_{x x} \sigma_{x y}}} \pm \frac{e^{i \beta_{y} z}}{\sigma_{y x} \sqrt{\sigma_{y x} \sigma_{y y}}}, \\
& B_{ \pm}=a\left(\frac{e^{i \beta_{x} z}}{\sigma_{x y} \sqrt{\sigma_{x x} \sigma_{x y}}} \pm \frac{e^{i \beta_{y} z}}{\sigma_{y y} \sqrt{\sigma_{y x} \sigma_{y y}}}\right) .
\end{aligned}
$$

The parameter $a$ in Eq. (73) adjusts the core shape of the initial vortex at the $z=0$ plane. It is an independent parameter that can be inserted into the solutions (50) and (51) as the amplitude factor.

The vortex state in each of the $E_{+}$and $E_{-}$components can be described in terms of the vectors fields $\psi_{+}=\nabla_{\perp} E_{+}$and $\psi_{-}=\nabla_{\perp} E_{-}[23,24]$ that characterise the local phase distributions. The mathematical approach is based on the parameters similar to the Stokes parameters used for the polarisation state of the beam:

$$
\begin{aligned}
& S_{0}^{( \pm)}=\left|\nabla_{\perp} E_{ \pm}\right|^{2}, \quad S_{1}^{( \pm)}=\left|\partial_{x} E_{ \pm}\right|^{2}-\left|\partial_{y} E_{ \pm}\right|^{2}, \\
& S_{2}^{( \pm)}=\partial_{x} E_{ \pm} \partial_{y} E_{ \pm}^{*}+\partial_{x} E_{ \pm}^{*} \partial_{y} E_{ \pm}, \\
& S_{3}^{( \pm)}=i\left(\partial_{x} E_{ \pm}^{*} \partial_{y} E_{ \pm}-\partial_{x} E_{ \pm} \partial_{y} E_{ \pm}^{*}\right)
\end{aligned}
$$

However, the above parameters characterise the vortex shape rather than the polarisation state. Deformation of the vortex core is described by the normalised parameter $S_{3}^{( \pm)}$ in the form

$$
\ell_{z}^{( \pm)}=\frac{i\left(\partial_{x} E_{ \pm}^{*} \partial_{y} E_{ \pm}-\partial_{x} E_{ \pm} \partial_{y} E_{ \pm}^{*}\right)}{\left|\nabla_{\perp} E_{ \pm}\right|^{2}} .
$$

In case of a simple vortex beam bearing the elliptically deformed and singly charged vortex at the axis, the value $\ell_{z}^{( \pm)}$characterises the orbital angular momentum of the beam. 
In the more complex case the parameter $\ell_{z}^{( \pm)}$describes the state of the vortex core: the modulus $\left|\ell_{z}^{( \pm)}\right|$is the ellipticity of the vortex core, whereas its sign gives the sign of the vortex topological charge. The periodical curves plotted in Fig. 6a refer to conversion of the vortex ellipticity both in the $E_{+}$and $E_{-}$components. The sharp peaks on the curves correspond to alternation of positive and negative vortex topological charges. The beat length is defined by the simple relation $\Lambda=\lambda /\left|n_{x}-n_{y}\right|$, where $\lambda$ is the wavelength in vacuum. In our case, it is about $\Lambda \approx 3.15 \mu \mathrm{m}$. The conversion peaks in the $E_{+}$and $E_{-}$ components are shifted relative to each other at the distance $l=\Lambda / 2$. What is important, the vortex conversion occurs within very small range of crystal lengths, a fraction of the wavelength. Fig. $6 \mathrm{~b}$ illustrates the shape of the vortex conversion line. Its linewidth is about $\Delta l \approx 0.25 \mu \mathrm{m}$. The peaks of the vortex conversion match with the transformation of the polarisation states from the right-hand to left-hand and vice-versa, i.e. the sign of the vortex charge and the handedness of the polarisation state change synchronically. In case of the plane wave, such a polarisation transformation is accompanied by the total energy transfer from one field component to another so that the vortex conversion cannot be observed in principle. However, the finite width of the singular beams results in the spatial field depolarisation both in the vicinity of the vortex core and for the beam as a whole. It is the depolarisation process that enables one to observe the vortex conversion.


Fig. 6. Periodic conversion of vortex ellipticity along the crystal length $z$ (in $\mathrm{cm}$ ) in the $E_{+}$and $E_{-}$components (a) and shape of the vortex-conversion line for $w_{x}=2 w_{y}=10 \mu \mathrm{m}, n_{x}=1.5, n_{y}=1.7$ and $a=0.6$ (b).

The conversion process occurs non-instantly but is accompanied by a chainlet of topological reactions. Typical pattern of those reactions is tracked with the aid of evolution of the phase distribution shown in Fig. 7. The regular phase spiral depicted in the first pattern of Fig. 7 characterises nearly ideal state of the positively charged centred vortex. The vortex charge sign is described by the handedness of the arrow in the figure. Perturbation of the phase spiral is caused by the two topological dipoles born on the dotted line in the next figure. One of the newborn vortices comes nearer to the centred 
vortex, causing phase unfolding. Then the topological dipole is again nucleated near the centre when the beam is transmitting, the positively charged vortex remaining at the centre while the negatively charged one being attracted to the other off-axis vortex to annihilate. The phase pattern in the vicinity of the beam axis remains nearly regular, up to the next cycle of the vortex conversion. The length of the conversion cycle is very short, being about $\delta z \approx 0.32 \mu \mathrm{m}$.

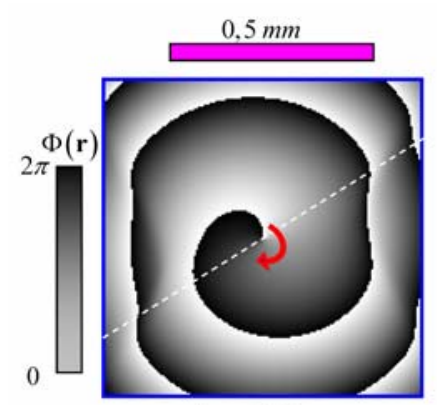

$\Delta z=0.38 \mu m$



$\Delta z=0.61 \mu m$

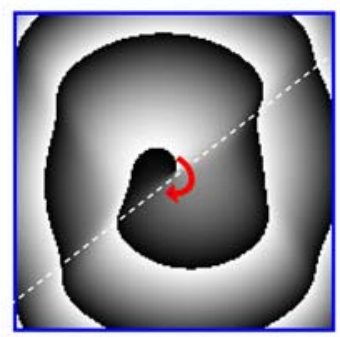

$\Delta z=0.49 \mu m$



$\Delta z=0.68 \mu m$

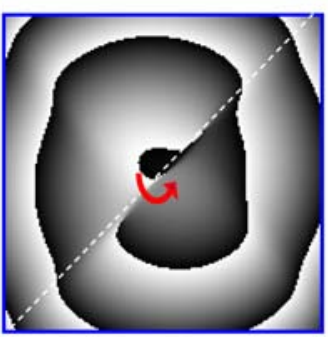

$\Delta z=0.54 \mu m$

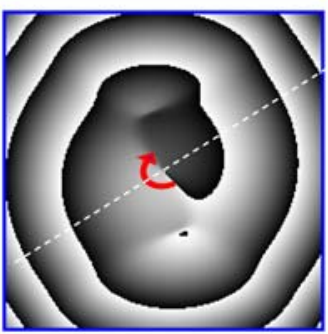

$\Delta z=0.70 \mu m$

Fig. 7. Evolution of phase distribution $\Phi(\mathbf{r})$ for the $E_{+}$component near the beam axis $r=0$ along the crystal length $z=\bar{z}+\Delta z$ in the area where the vortex is transformed (the beam and crystal parameters are indicated in Fig. 6 and $\bar{z}=11.656 \mathrm{~mm}$ ).

Perturbations of the polarisation state at the beam cross section for account of the elliptical deformation of the extraordinary beam imply spatial light depolarisation and descent of the amplitude value of the spin angular momentum. Phase difference along the axial direction between the ordinary and extraordinary plane waves results in oscillations of the polarisation state from the right-hand to the left-hand one. However, the other plane waves in the angular spectra of the $E$ - and $H$-beams acquire different values of the elliptic polarisation, so that the beam as a whole is depolarised. The spin angular momentum of the paraxial beam in uniaxial crystals can be calculated as [25]

$$
S_{z}=\frac{4 \operatorname{Im}\left(\int_{-\infty}^{\infty} E_{x} E_{y}^{*} d x d y\right)}{\int_{-\infty}^{\infty}\left(\left|E_{x}\right|^{2}+\left|E_{y}\right|^{2}\right) d x d y},
$$

while the polarisation degree is defined as 


$$
P=\frac{2\left|\int_{-\infty}^{\infty} E_{x} E_{y}^{*} d x d y\right|}{\int_{-\infty}^{\infty}\left(\left|E_{x}\right|^{2}+\left|E_{y}\right|^{2}\right) d x d y} .
$$
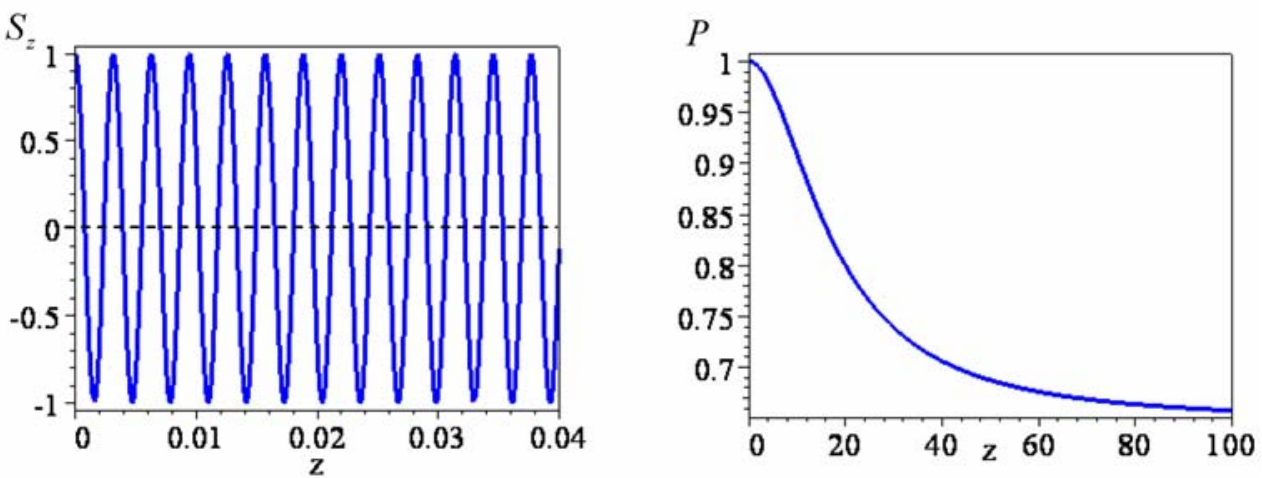

Fig. 8. Evolutions of (a) spin angular momentum $S_{z}$ and (b) polarisation state $P$ along the crystal length $z$ (in $\mathrm{mm}$ ) for the vortex beam with the parameters indicated in Fig. 6.

The results of our computer simulation of the $S_{z}(z)$ and $P(z)$ parameters are shown in Fig. 8. The oscillations of the spin angular momentum $S_{z}$ from +1 to -1 are typical only in the initial part of crystal length. The positions of spin angular momentum changes coincide with those of the vortex sign conversion and have the same beating length $\Lambda$. When the crystal length increases, the amplitude of the oscillation comes gradually down to some utmost value unequal to zero. The envelope of the spin angular momentum oscillations is the polarisation degree $P$. The utmost value of $P(z)$ depends on the beam waists $w_{x}$ and $w_{y}$, and on the refractive indices of the crystal.

\section{Discussion and conclusions}

When analysing the beam propagation along the direction perpendicular to the optic axis of the uniaxial crystal, we have derived the corresponding equations and found their solutions in the form of the generalised Hermite-Gaussian beams. By manipulating these beams, we have constructed the generalised Laguerre-Gaussian beams at the $z=0$ plane and analysed their evolution in homogeneous isotropic media. We have shown that the standard elliptic Laguerre-Gaussian beam bearing the centred high-order optical vortex at the initial plane $z=0$ is destroyed when propagating in such a way that the centred highorder vortex is split into a row of singly charged vortices, while the ring dislocations are split into a series of topological dipoles. All the vortices in this construction get mixed, thus forming a complex combined singular beam. At the far field, the ring dislocations recover a nearly regular structure, while the singly charged vortices near the beam axis are not united into the centred high-order optical vortex at any beam length. 
We have traced propagation of the circularly polarised standard elliptic LaguerreGaussian beam with the zero radial index $n=0$, bearing the $m$-charged centred vortex along the crystal, the ellipse axis of the beam cross section being directed along the major directions of the permittivity tensor. We have shown that the additional elliptic deformation of the extraordinary beam results in topological reactions that cannot favour recovering the initial singular structure. The degenerated centred vortex decays into a row of singly charged vortices when shifting slightly from the $z=0$ plane, as it takes place in free space. However, the additional geometrical perturbations in the extraordinary beam result in different rotation rates of the perturbed vortex rows in the ordinary and extraordinary beams. In the long run, the form of the resulting beam is radically distorted at far field. The beam loses its initial elliptic shape, while the conoscopic pattern in the form of distorted hyperbolic lines paints the beam components up to irrecognizability. It is such a complex picture of the field distribution in each circularly polarised component that causes spatial depolarisation of the vector singular beam.

We have predicted conversion of the vortex topological charge in the singly charged elliptic vortex beam, similar to that in the astigmatic lens [12] or optical fibres [26]. However, there is a radical difference in these processes. First of all, the vortex conversion in the astigmatic lenses or optical fibres is associated either with different curvatures of the lens surfaces along the major astigmatic axes or variations of the refractive index of the fibre cross section. In our case we deal with a homogenous medium. Nonetheless, the symmetry of the optical birefringence in crystals causes a symmetry breakdown in the extraordinary beam that spreads through the crystal changing the scale along one of the crystallographic axes. At the same time, the resulting elliptic deformation of the extraordinary beam provokes a perturbation of its helix wavefront. The destructive interference between the unperturbed ordinary beam and the perturbed extraordinary beams entails a chainlet of topological reactions in the vicinity of the beam axis and a periodic process of vortex conversion at the beam axis.

In the second place, the singular beam with the converted vortex can propagate along indefinitely large distances after the astigmatic lens while the life-length of the converted beam in the crystal is considerably less or comparable with the light wavelength. This is conditioned by the wavefront shape of the perturbed extraordinary beam. By all appearances, it is the reason why this drastic phenomenon has not been revealed earlier.

Different field distributions in the ordinary and extraordinary beams are also associated with the peculiarities of spin angular momentum of the singular beam. The oscillations of the spin angular momentum of the vortex beam presented in this study are accompanied by spatial depolarisation of the field that decreases the spin angular momentum amplitude. Notice that in case when the beam propagates along the optic axis of crystal, any transformation of the spin angular momentum is compensated by the orbital angular momentum in the form of the optical vortex generation, so that the sum of the spin angular momentum and the orbital angular momentum is conserved. The axial symmetry of the crystal in this direction enables dividing the spin angular and orbital angular mo- 
mentums from the mechanical angular momentum of the crystal medium, so that any transformation of the spin angular momentum has an immediate impact on the orbital angular momentum, without implicating the crystal medium in the process. For example, a small beam inclination relative to the optic axis entails a non-local lateral shift of the beam, in order to compensate the access of the orbital angular momentum [27], while the mechanical angular momentum of the medium is not involved.

The beam propagation along the directions perpendicular to the optic axis breaks the former symmetry. Naturally, the vortex conversion in this case results in transformations of the orbital angular momentum. But now the three processes take part in the phenomenon. The spin angular and orbital angular momentums are supplemented by the response of the crystal medium. It is the sum of these three processes that must reduce to conservation of the total angular momentum. Thus, the contribution of the vortex conversion to these three processes needs more detailed theoretical and experimental investigations.

A peculiar question concerns the periodically repeated conversion of the centred vortex sign. This process is synchronised with the conversion of the spin angular momentum. From our viewpoint, synchronisation of these processes is associated with a phase match between the $E_{x}$ and $E_{y}$ components of the beam. The conversion of handedness of the circular polarisation occurs when the $E_{x}$ and $E_{y}$ components have the antiphase state. However the vortex conversion near the beam axis is caused by a destructive interference that requires also the antiphase state of the $E_{x}$ and $E_{y}$ components. When the beam waist is sufficiently large, almost all the energy is transferred from the right circularly polarised component into the left one under the conditions of the above match, so that we have $E_{x} \approx E_{y}$ and the vortex conversion cannot be observed in experiment. However, the evolution of the spin angular momentum along the crystal length entails spatial depolarisation of light. There is no complete energy transfer between the beam components at the beam cross section. It is this circumstance that should enable us to detect the vortex conversion effect in the experiment.

\section{Acknowledgement}

One of the authors (A. V.) thanks E. Abramochkin for fruitful discussions on the mathematical background of generalised Hermite-Laguerre-Gaussian beams.

\section{References}

1. Born M and Wolf E, Principles of optics. New York: Pergamon (1975).

2. Fleck $\mathbf{J}$ and Felt $\mathrm{M}, 1983$. Beam propagation in uniaxial anisotropic media. J. Opt. Soc. Am. 73: 920-926

3. Seshadri S R, 2003. Basic elliptical Gaussian wave and beam in a uniaxial crystal. J. Opt. Soc. Am. A 20: 1818-828

4. Ciattoni A and Palma C, 2003. Optical propagation in uniaxial crystals orthogonal to the optical axis: paraxial theory and beyond. J. Opt. Soc. Am. A 20: 2163-2171. 
5. Soskin MS and Vasnetsov MV, 2001. Singular optics. Progress in Optics, 42: 219276.

6. Fadeyeva T A, Rubass A F, Sokolenko B V and Volyar A V. 2009. The vortex-beam "precession" in a rotating uniaxial crystal. J. Opt. A: Pure Appl. Opt. 11: 094008.

7. Volyar A, Shvedov V and Fadeyeva T, 2001. Structure of nonparaxial Gaussian beams near the focus: 2. Optical vortices. Opt. Spectr. 90: 104-112.

8. Kotlyar V, Khonina S, Almazov A, Soifer V, Jefimovs K and Turunsen J, 2006. Elliptic Laguerre-Gaussian beams. J. Opt. Soc. Amer. A. 23: 43-56.

9. Wada A, Ohtani T, Miyamoto Y and Takeda M, 2005. Propagation analysis of the Laguerre-Gaussian beam with astigmatism. J. Opt. Soc. Amer. A. 22: 2746-2755.

10. Du X and Zhao D, 2006. Propagation of decentered elliptical Gaussian beams in apertured and nonsymmetrical optical systems. J. Opt. Soc. Am. A. 23: 625-631.

11. Dennis M R, 2006. Row of optical vortices from elliptically perturbing a high order beam. Opt. Lett. 31: 1325-1327

12. Bekshaev A, Soskin M and Vasnetsov M, 2003. Optical vortex symmetry breakdown and decomposition of the orbital angular momentum of light beams. J. Opt. Soc. Amer. A. 20: $1635-1643$

13. Bekshaev A Ya, Soskin MS and Vasnetsov MV, 2004. Transformation of higherorder optical vortices upon focusing by an astigmatic lens. Opt. Commun. 241: 237247

14. Bekshaev A Ya and Karamoch AI, 2008. Astigmatic telescopic transformation of high-order optical vortex. Opt. Commun. 281: 5687-5696

15. Wünsche A, 1989. Generalized Gaussian beam solution of paraxial optics and their connection to a hidden symmetry. J. Opt. Soc. Am. A. 6: 1320-1329

16. Abramochkin E, Razueva E and Volostnikov V, 2010. General astigmatic transform of Hermite-Laguerre-Gaussian beams. J. Opt. Soc. Am. A, 27: 2506-2513

17. Abramochkin E and Volostnikov V, 2004. Generalized Gaussian beams. J. Opt. A: Pure Appl. Opt., 6: S157-S161

18. Abramochkin E and Volostnikov V, 2010. Generalized Hermitte-Laguerre-Gaussian beams. Physics of Wave Phenomena, 18: 14-22

19. Zeuderer E, 1986. Complex argument Hermite-Gaussian and Laguerre-Gaussian beams. J. Opt. Soc. Am. A. 3: 465-469

20. Bandres M and Cutierrez-Vega J, 2008. Elliptical beams. Opt. Express. 10: $21087-$ 21092

21. Abramochkin E, Private communication.

22. Nye J,F, Natural focusing and fine structure of light: Caustics and wave dislocations, Bristol: Institute of Physics Publishing, (1999).

23. Egorov YuA, Fadeyeva TA and Volyar AV, 2004. The fine structure of singular beams in crystals: colours and polarization. J. Opt. A: Pure Appl. Opt. 6: S217-S228.

24. Dennis M R, 2004. Local phase structure of wave dislocation lines: twist and twirl. J. Opt. A: Pure Appl. Opt. 6: S202 - S208.

Ukr. J. Phys. Opt. 2011, V12, №2 
25. Fadeyeva TA and Volyar AV, 2010. Extreme spin-orbit coupling in crystal-traveling paraxial beams. J. Opt. Soc. Am. A. 27: 381-389.

26. Alexeyev CN, Volyar AV and Yavorsky MA, 2007. Fiber optical vortices. Lasers, optics and electro-optics research trends, ed. L. I. Chen. - New York: Nova Publishers, 131-223.

27. Fadeyeva TA, Rubass AF and Volyar AV, 2009. Transverse shift of a high-order paraxial vortex-beam induced by a homogeneous anisotropic medium. Phys. Rev. A. 79: 053815-1-12.

Fadeyeva T., Alexeyev C., Sokolenko B., Kudryavtseva M. and Volyar A., 2011. Non-canonical propagation of high-order elliptic vortex beams in a uniaxially anisotropic medium Ukr.J.Phys.Opt. 12: 62-82.

Анотація. В роботі проаналізовано поширення параксіальних і не параксіальних променів у напрямку перпендикулярному до оптичної осі одновісного анізотропного середовища (безмежсний оптично одновісний кристал). Запропоновано параксіальний розв'язок у виглядi загальних променів Ерміта-Гауса, які поширюються перпендикулярно до оптичної осі в одновісних анізотропних середовищах. Також побудовані промені Лагерра-Гауса для площуини z=0 і проаналізована їх еволючія в однорідному ізотропному середовищі. Порівнюючи ї̈ з еволюиією стандартних променів Лагерра-Гауса з $n=0$ та $m \neq 0$ в кристалах виявлено, що додаткова еліптична деформачія незвичайного променя приводить до топологічної реакиії, що суттєво змінює структуру поля, при врахуванні різних швидкостей обертання низки вихорів, які походять від центрованого виродженого оптичного вихору $і$ коноскопічної картини. Передбачено конверсію топологічного заряду вихору на осі променя, подібну до властивої для астигматичних лінз і проаналізовані відмінності в обох випадках. Продемонстрована можливість синхронних осцилящій спінового кутового моменту $і$ знаку топологічного заряду вихора на осі променя. 